\title{
Ultrastructural and Immunocytochemical Analysis of Multilineage Differentiated Human Dental Pulp- and Umbilical Cord-Derived Mesenchymal Stem Cells
}

\author{
T. Struys $^{a} \quad$ M. Moreels ${ }^{b} \quad$ W. Martens ${ }^{a} \quad$ R. Donders ${ }^{a} \quad$ E. Wolfs ${ }^{a} \quad$ I. Lambrichts ${ }^{a}$ \\ ${ }^{\mathrm{a}}$ Lab of Histology, Biomedical Research Institute, Hasselt University, Diepenbeek, and ${ }^{\mathrm{b}}$ Molecular and Cellular Biology, \\ Institute for Environment, Health and Safety, Belgian Nuclear Research Centre (SCK-CEN), Mol, Belgium
}

\section{Key Words}

Stem cells $\cdot$ Mesenchyme $\cdot$ Differentiation $\cdot$ Dental pulp $\cdot$

Umbilical cord • Ultrastructure $\cdot$ Adipogenesis ·

Osteogenesis · Chondrogenesis

\begin{abstract}
Mesenchymal stem cells (MSCs) are one of the most promising stem cell types due to their availability and relatively simple requirements for in vitro expansion and genetic manipulation. Besides the well-characterized MSCs derived from bone marrow, there is growing evidence suggesting that dental pulp and the umbilical cord matrix both contain a substantial amount of cells having properties similar to those of MSCs. In order to assess the potential of dental pulp-derived MSCs (DPSC) and umbilical cord-derived MSCs (UCSC) in future clinical applications, it is essential to gain more insight into their differentiation capacity and to evaluate the tissues formed by these cells. In the present study, the morphological and ultrastructural characteristics of DPSC and UCSC induced towards osteogenic, adipogenic, and chondrogenic lineages were investigated. Cultured DPSC and UCSC showed a similar expression pattern of antigens characteristic of MSCs including CD105, CD29, CD44, CD146, and STRO-1. Under appropriate culture conditions, both DPSC and UCSC showed chondrogenic and osteogenic potential.
\end{abstract}

T. Struys and M. Moreels contributed equally to this work.
Adipogenesis could be only partially induced in DPSC resulting in the de novo expression of fatty acid binding protein (FABP), whereas UCSC expressed FABP combined with a very high accumulation of lipid droplets in the cytoplasm. Our results demonstrate, at the biochemical and ultrastructural level, that DPSC display at least bilineage potential, whereas UCSC, which are developmentally more primitive cells, show trilineage potential. We emphasize that transmission electron microscopical analysis is useful to elucidate detailed structural information and provides indisputable evidence of differentiation. These findings highlight their potential therapeutic value for cell-based tissue engineering.

Copyright $\odot 2010$ S. Karger AG, Basel

\section{Abbreviations used in this paper}

DMEM-LG Dulbecco's modified Eagle's medium with GlutaMAX-I and low glucose

DPSC dental pulp stem cell

$F \quad$ fungizone

FABP fatty acid binding protein

FBS fetal bovine serum

MSC mesenchymal stem cell

ORO Oil red O staining

$\mathrm{P} \quad$ penicillin

PBS phosphate-buffered saline

RER rough endoplasmatic reticulum

S streptomycin

UCSC umbilical cord stem cell

\section{KARGER}

Fax +4161306 1234 E-Mail karger@karger.ch www.karger.com
(C) 2010 S. Karger AG, Base

$1422-6405 / 11 / 0000-0000 \$ 38.00 / 0$

Accessible online at:

www.karger.com/cto
Drs. Tom Struys

Lab of Histology, Biomedical Research Institute

Hasselt University, Campus Diepenbeek, Agoralaan, Building C, Office C008

BE-3590 Diepenbeek (Belgium)

Tel. +32 112692 21, Fax +32 112685 99, E-Mail tom.struys@ uhasselt.be 


\section{Introduction}

Mesenchymal stem cells (MSCs) are self-renewing and multipotent cells which were first isolated from bone marrow [Friedenstein et al., 1976]. MSCs represent a heterogeneous and typical fibroblast-like cell population [Prockop, 1997] which has been described to have the ability to differentiate into multiple mesenchymal cell types such as adipocytes, osteoblasts, and chondroblasts [Pittenger et al., 1999; Jiang et al., 2002]. MSCs have generated a great deal of interest for their potential application in regenerative medicine and tissue engineering [Caplan, 2007]. Due to their extensive expansion rate and differentiation potential, MSCs are among the first stem cell types that possess the features necessary to be introduced into the clinical environment. In addition, their hypoimmunogenic and even immunosuppressive characteristics [Uccelli et al., 2007] show that they may be extremely suitable for allogeneic cell transplantation. Currently, bone marrow represents the main source of MSCs for both experimental and clinical studies [Giordano et al., 2007; Lee et al., 2008]. However, harvesting bone marrow is a highly invasive procedure with a considerable risk of donor site morbidity. Moreover, only a limited number of MSCs can be isolated from the collected tissue and the number and differentiation potential of human bone marrow-derived MSCs significantly decreases with age [Mueller and Glowacki, 2001; Stenderup et al., 2003]. Therefore, the search for alternative MSC sources is of significant value. Surgical and biological waste material might provide ideal donor tissue for isolating stem cells. Dental pulp isolated from third-molar and umbilical cord samples which are readily available following surgical interventions or delivery, respectively, might be new promising sources.

Dental pulp is the mucoid connective tissue that occupies the central space in the tooth. This tissue is completely encased within the mineralized dentin, which is produced by the odontoblasts, a postmitotic cell type located on the periphery of the pulp tissue. The core of the pulp tissue is composed of fibroblast-like cells producing a meshed network of collagen and elastin fibers which is embedded in an amorphous ground substance rich in proteoglycans and glycosaminoglycans [Linde, 1985]. On the other hand, the umbilical cord is composed of an embryonic mucoid connective tissue matrix, called Wharton's jelly, located between the covering amniotic epithelium and the umbilical vessels. The matrix consists of myofibroblast-like stromal cells and collagen fibers embedded in an amorphous ground substance rich in proteoglycans [Kobayashi et al., 1998; McElreavey et al., 1991].

Recent studies have shown the MSC nature of cells within both mucoid connective tissues [Gronthos et al., 2000, 2002; Mitchell et al., 2003; Shi and Gronthos, 2003; Weiss et al., 2003; Wang et al., 2004]. These cells share similar characteristics with bone marrow-derived MSCs including adherence to plastic substrates, surface marker expression, and differentiation potential into lineages of mesenchymal tissues such as bone, cartilage, and adipose tissue [Gronthos et al., 2002; Mitchell et al., 2003; Shi and Gronthos, 2003; Wang et al., 2004; Carlin et al., 2006; Weiss et al., 2006; Takeda et al., 2008]. In order to assess the potential of dental pulp stem cells (DPSC) and umbilical cord stem cells (UCSC) for future clinical applications, it is essential to further explore the lineage-specific tissues formed by these stem cells. Although there are a number of papers describing their characteristics, structural and ultrastructural descriptions of their phenotype are still lacking. To fill this gap, the present study was aimed at providing an in-depth morphological description of both DPSC and UCSC induced towards adipogenic, osteogenic, and chondrogenic lineages.

\section{Materials and Methods}

Cell Culture Media and Chemicals

Dulbecco's modified Eagle's medium with GlutaMAX ${ }^{\mathrm{TM}}-\mathrm{I}$ and low glucose (DMEM-LG), Basal Medium Eagle (BME), $\alpha$-MEM, DMEM-F12, penicillin (P), streptomycin (S), fungizone (F), and L-glutamine were obtained from GIBCO Invitrogen Corp. (Paisley, UK). Fetal Bovine Serum (FBS) was obtained from Biochrom AG (Berlin, Germany). All other chemicals were purchased from Sigma Chemical Co. (St. Louis, Mo., USA).

Isolation and Culture of DPSC

Human dental pulp tissue was isolated from third molars that were extracted for therapeutic or orthodontic reasons with the patients' informed consent $(\mathrm{n}=10)$. Immediately after extraction, from each patient, 4 teeth from each patient were fractured mechanically and the pulp tissue was removed by means of a forceps. Tissue was collected in BME supplemented with heat-inactivated $10 \%$ FBS, $2 \mathrm{mM}$ L-glutamine, $50 \mu \mathrm{G} / \mathrm{ml}$ ascorbic acid, $1 \% \mathrm{P}, 1 \% \mathrm{~S}$, and $0.2 \% \mathrm{~F}$, and then transferred to the laboratory for cell isolation. After washing in the same medium to remove any contaminating blood, the pulp tissue was minced into small fragments $\left(1-2 \mathrm{~mm}^{3}\right)$. These explants were transferred to 6 -well plates containing the same medium as described above. The explants were left undisturbed for 14 days to allow the migration of cells from the explants. Cells were cultured at $37^{\circ} \mathrm{C}$ in a humidified atmosphere containing $5 \% \mathrm{CO}_{2}$, and the medium was changed every 2 days. After 2 weeks, the explants were discarded from the culture dish and the resulting cell population was serially passaged and refed every 2 days. The cells used in the study were between P2 and P5. 


\section{Isolation and Culture of UCSC}

Human umbilical cords were obtained from full-term deliveries, with the mothers' informed consent, and processed within $24 \mathrm{~h}(\mathrm{n}=10)$. The cords were immersed in a sterile vessel containing $0.01 \mathrm{M}$ phosphate-buffered saline (PBS; $\mathrm{pH}$ 7.2) supplemented with $1 \% \mathrm{P}, 1 \% \mathrm{~S}$, and $0.2 \% \mathrm{~F}$ and then transferred to the laboratory for further cell isolation. After washing in PBS to remove any contaminating blood, the cord was cut into small pieces $(1-2 \mathrm{~cm}$ in length). Amniotic membrane, umbilical arteries, and veins were removed, and the remaining tissue was minced into small fragments $\left(1-2 \mathrm{~mm}^{3}\right)$. The tissue pieces were transferred to 6-well plates containing DMEM-LG supplemented with $10 \% \mathrm{FBS}+1 \%$ $\mathrm{P}, 1 \% \mathrm{~S}$, and $0.2 \% \mathrm{~F}$, and incubated at $37^{\circ} \mathrm{C}$ in a humidified atmosphere containing $5 \% \mathrm{CO}_{2}$. The explants were left undisturbed for 14 days to allow the migration of cells from the explants. They were refed and passaged when necessary. The cells used in the study were between P2 and P5.

\section{Differentiation Procedures}

The adipogenic, osteogenic, and chondrogenic differentiation capabilities of both DPSC and UCSC were tested by incubating them in differentiation media from the hMSC Functional Identification Kit (SC006; R\&D Systems, UK).

\section{Adipogenic Differentiation Protocol}

Following trypsinization, DPSC and UCSC were seeded onto $12-\mathrm{mm} \varnothing$ glass coverslips for light microscopy or onto plastic coverslips (Thermanox ${ }^{\circledR}$; Electron Microscopy Sciences) for electron microscopy at a density of $3.7 \times 10^{4}$ cells per well in $\alpha$-MEM basal medium supplemented with $10 \% \mathrm{FBS}+1 \% \mathrm{P}, 1 \% \mathrm{~S}, 0.2 \% \mathrm{~F}$, and $2 \mathrm{mM} \mathrm{L-glutamine.} \mathrm{Cells} \mathrm{were} \mathrm{cultured} \mathrm{at} 37^{\circ} \mathrm{C}$ in a humidified atmosphere containing $5 \% \mathrm{CO}_{2}$. When confluence was reached, the medium was changed to the adipogenic differentiation medium (R\&D Systems). The medium was changed every 3 days. After 3 weeks, cells seeded on glass coverslips were washed with PBS and fixed with $4 \%$ formaldehyde (Unifix, Duiven, The Netherlands) for $20 \mathrm{~min}$ at room temperature. Cells seeded on plastic coverslips were fixed with $2 \%$ glutaraldehyde in $0.05 \mathrm{M}$ cacodylate buffer ( $\mathrm{pH} 7.3$ ) overnight at $4^{\circ} \mathrm{C}$.

\section{Osteogenic Differentiation Protocol}

Following trypsinization, DPSC and UCSC were seeded onto $12-\mathrm{mm} \emptyset$ glass coverslips for light microscopy or onto plastic coverslips (Thermanox; Electron Microscopy Sciences) for electron microscopy coated with fibronectin (R\&D Systems) with a density of $7.4 \times 10^{3}$ cells per well in $\alpha$-MEM basal medium supplemented with $10 \% \mathrm{FBS}+1 \% \mathrm{P}, 1 \% \mathrm{~S}, 0.2 \% \mathrm{~F}$, and $2 \mathrm{mM} \mathrm{L}$-glutamine. Cells were cultured at $37^{\circ} \mathrm{C}$ in a humidified atmosphere containing $5 \% \mathrm{CO}_{2}$. When $50-70 \%$ confluence was reached, the medium was changed to the osteogenic differentiation medium (R\&D Systems). The medium was changed every 3 days. After 3 weeks, cells seeded on glass coverslips were washed with PBS and fixed with $4 \%$ formaldehyde (Unifix) for $20 \mathrm{~min}$ at room temperature. Cells seeded on plastic coverslips were fixed with $2 \%$ glutaraldehyde in $0.05 \mathrm{M}$ cacodylate buffer ( $\mathrm{pH} 7.3$ ) overnight at $4^{\circ} \mathrm{C}$.

Chondrogenic Differentiation Protocol

Following trypsinization, DPSC and UCSC were seeded in $15-$ $\mathrm{ml}$ conical tubes with a density of $25 \times 10^{4}$ cells per tube in DMEM-F12 basal medium, $1 \%$ insulin, transferrin and selenium supplement (R\&D Systems) + 1\% P, 1\% S, 0,2\% F, and $2 \mathrm{~mm} \mathrm{L-}$ glutamine. Cells were centrifuged for $5 \mathrm{~min}$ at $1,200 \mathrm{rpm}$ and subsequently resuspended in chondrogenic differentiation medium (R\&D Systems). Afterwards, cells were centrifuged again for $5 \mathrm{~min}$ at $1,200 \mathrm{rpm}$ and subsequently cultured as pellets at $37^{\circ} \mathrm{C}$ in a humidified atmosphere containing $5 \% \mathrm{CO}_{2}$. The medium was changed every 3 days. After 3 weeks, cell spheres were washed with PBS and fixed with either $4 \%$ formaldehyde (Unifix) for 20 min at room temperature for light microscopical analysis or with $2 \%$ glutaraldehyde in $0.05 \mathrm{M}$ cacodylate buffer ( $\mathrm{pH}$ 7.3) overnight at $4^{\circ} \mathrm{C}$ for electron microscopy.

\section{Histochemical Stainings}

Differentiation of cells into the adipogenic, osteogenic, and chondrogenic lineages was determined using different histological staining techniques. Adipogenic differentiation was evaluated using Oil red O (ORO) to reveal lipid droplet accumulation in the cell cytoplasm. Osteogenic differentiation was demonstrated using Alizarin red S staining to detect calcified deposits in the cell cultures. Finally, Alcian blue staining was used to reveal chondrogenic differentiation showing the production of a ground substance matrix. In all stainings, nuclei were counterstained using Mayer's hematoxylin. After mounting in an aqueous mounting medium (Aquatex; Merck, Darmstadt, Germany) stained cells were examined using a photomicroscope equipped with an automated digital camera (Nikon Eclipse 80i; Nikon Co., Japan).

\section{Immunocytochemistry}

Immunocytochemical stainings were performed using the peroxidase-based EnVision System ${ }^{\circledR}$ (DakoCytomation, Glostrup, Denmark). After fixation and washing in $0.01 \mathrm{M} \mathrm{PBS}(\mathrm{pH}$ 7.2), cells were permeabilized when needed with $0.05 \%$ Triton X-100 (Boehringer, Mannheim, Germany) in PBS for $30 \mathrm{~min}$ at $4^{\circ} \mathrm{C}$ prior to labeling and then washed again with PBS. Nonspecific binding sites were subsequently blocked with $3 \%$ normal goat or donkey serum (depending on the secondary antibody that was used) (DakoCytomation) in PBS for 20 min. After washing, cells were incubated with mouse monoclonal antibodies against CD29 (1:35; Abcam, Cambridge, UK), CD44 (1:200; Abcam), CD105 (1:1,000; Abcam), STRO-1 (1:50, R\&D Systems), vimentin (1:500; DakoCytomation), and nestin (1:500, Chemicon, USA), or osteocalcin (1:100, R\&D Systems) rabbit polyclonal antibody against CD146 (ready to use; Abcam), or goat polyclonal antibodies against fatty acid binding protein (FABP)-4 (1:100, R\&D Systems), and aggrecan (1:100, R\&D Systems) for $1 \mathrm{~h}$. The cells were then washed again and incubated for $30 \mathrm{~min}$ with goat anti-mouse, goat anti-rabbit, or donkey anti-goat horseradish peroxidase-conjugated secondary antibodies. A high-sensitivity diaminobenzidine chromogenic substrate system and Mayer's hematoxylin were respectively used to visualize the peroxidase and for counter staining. After mounting in an aqueous mounting medium (Aquatex; Merck, Darmstadt, Germany), cell-seeded coverslips were examined using a photomicroscope equipped with an automated camera (Nikon Eclipse 80i, Nikon). Control cells were subjected to the same immunoperoxidase staining, with omission of the primary antibody.

\section{Ultrastructural Analysis}

Following fixation, the fixative was gently aspirated with a glass pipette, and the cells were postfixed in $2 \%$ osmium tetroxide 
for $1 \mathrm{~h}$ and stained with $2 \%$ uranyl acetate in $10 \%$ acetone for 20 min. Subsequently, the cell-seeded coverslips were put through a dehydrating series of graded concentrations of acetone and embedded in araldite according to the pop-off method [Bretschneider et al., 1981]. Ultra-thin sections $(0.06 \mu \mathrm{m})$ were mounted on $0.7 \%$ formvar-coated grids, contrasted with uranyl acetate and lead citrate, and examined in a Philips EM 208 transmission electron microscope operated at $80 \mathrm{kV}$. Digital images were captured using a Morada camera system and analyzed using SIS analysis software (Germany).

\section{Image Analysis}

Images were acquired by 3 independent investigators that were blind for cell type and experimental conditions. For each of the experimental conditions, 50 microscopic fields were analyzed with $40 \times$ and 5,000 $\times$ magnifications for light and electron microscopy, respectively. The images shown in this article were selected as being representative of the indicated condition.

\section{Results}

\section{Morphology and Immunophenotype}

After 2 weeks, cells isolated from umbilical cord and dental pulp explants grew out of the explant and adhered to a plastic surface. The morphology of cultured DPSC and UCSC included mesenchymal-like cells with a polygonal or spindle-shaped appearance (fig. 1a, b). The morphology of the cells changed with a higher level of confluence; the cytoplasm became narrower, and the cells appeared mostly spindle shaped.

The characterization of markers expressed by the cells was performed by immunocytochemical analysis. Surface phenotyping revealed a similar expression pattern of antigens characteristic of MSCs including CD105+, CD29+, CD44+, CD146+, and STRO-1 (fig. 1c-1). Further evaluation of cytoskeletal elements showed that both HDPC and UCSC expressed vimentin (fig. 1m, n). Nestin expression could only be detected in DPSC (fig. 1o, p).

\section{Ultrastructural Analysis}

At the ultrastructural level, both DPSC and UCSC showed large euchromatic nuclei with prominent nucleoli. The cell surface often showed filopodia. The cell cytoplasm was characterized by distinct subcellular compartments including an organelle-rich perinuclear zone and a rather electron-lucent peripheral region (fig. 1q, $t$ ). The perinuclear region mainly contained numerous mitochondria, prominent rough endoplasmatic reticulum (RER) cisternae and some Golgi apparatus. Interestingly, the RER cisternae were distended and contained moderately electron-dense secretory material. In addition, the cytoplasm was rich in intermediate filaments. The pe- riphery of the cytoplasm exhibited a rather electron-lucent appearance due to the absence of organelles. However, this region contained some vacuoles, vesicles, and bundles of intermediate filaments. Thick arrays of thin filaments were commonly observed in close proximity to the cell edges. No extracellular matrix deposition or specialized cell junctions were observed (fig. 1q-v).

\section{Differentiation of Cells into Mesenchymal Lineages}

The differentiation potential of DPSC and UCSC was tested by performing a multilineage differentiation assay following the manufacturer's instructions.

ORO staining did not reveal any intracellular lipid droplets following the adipogenic differentiation of DPSC ORO, with the exception of $30 \%$ of the tests, in which a few ORO-positive cells were observed (fig. 2a, b). Nevertheless, immunocytochemical analysis revealed the expression of FABP, an adipocyte-specific gene, in the cells after adipogenic induction (fig. $2 c, d$ ). These findings were confirmed at the ultrastructural level. However, adipogenic-induced DPSC showed many elongated and branched mitochondria, extremely dilated RER, and numerous multilamellar vesicles (fig. $3 \mathrm{a}, \mathrm{b}$ ).

In contrast, UCSC grown in adipogenic medium formed intracellular lipid droplets (indicated by ORO staining) within 1 week; these enlarged over time during treatment (fig. 2e, f). Adipocytic phenotypes in induced cells were first signaled by the appearance of multisized,

Fig. 1. Morphology and immunophenotypic profile of human DPSC and UCSC. Human DPSC (a) and UCSC (b) both exhibited a typical fibroblast-like morphology. Cells showed adherence to glass and plastic substrates and were capable of forming colonies in vitro. The presence of several common MSC markers was tested to determine the immunophenotypic profile of both cell types. Human DPSC (c, e, g, i, $\mathbf{k}, \mathbf{m})$ and $\operatorname{UCSC}(\mathbf{d}, \mathbf{f}, \mathbf{h}, \mathbf{j}, \mathbf{I}, \mathbf{n})$ showed protein expression of CD29, CD44, CD105, CD146, STRO-1, and vimentin, respectively. Human DPSC (o) also expressed nestin in contrast to UCSC (p). q-v Ultrastructural analysis of human DPSC (q-s) and UCSC (t-v). Both cell types are characterized by large oval nuclei, predominantly euchromatic, with 1 or more prominent nucleoli. The cell cytoplasm is characterized by distinct subcellular compartments $(\mathbf{q}, \mathbf{t})$. The perinuclear organellerich zone contains mainly RER cisternae, elongated mitochondria, and some Golgi apparatus $(\mathbf{r}, \mathbf{u})$. The peripheral part of the cytoplasm exhibited a rather electron-lucent appearance due to the absence of organelles. Nevertheless, multiple small vacuoles and the expression of some intermediate filaments could be detected in this region $(\mathbf{s}, \mathbf{v})$. Neither specialized cell junctions nor the production of any kind of extracellular material could be observed. 

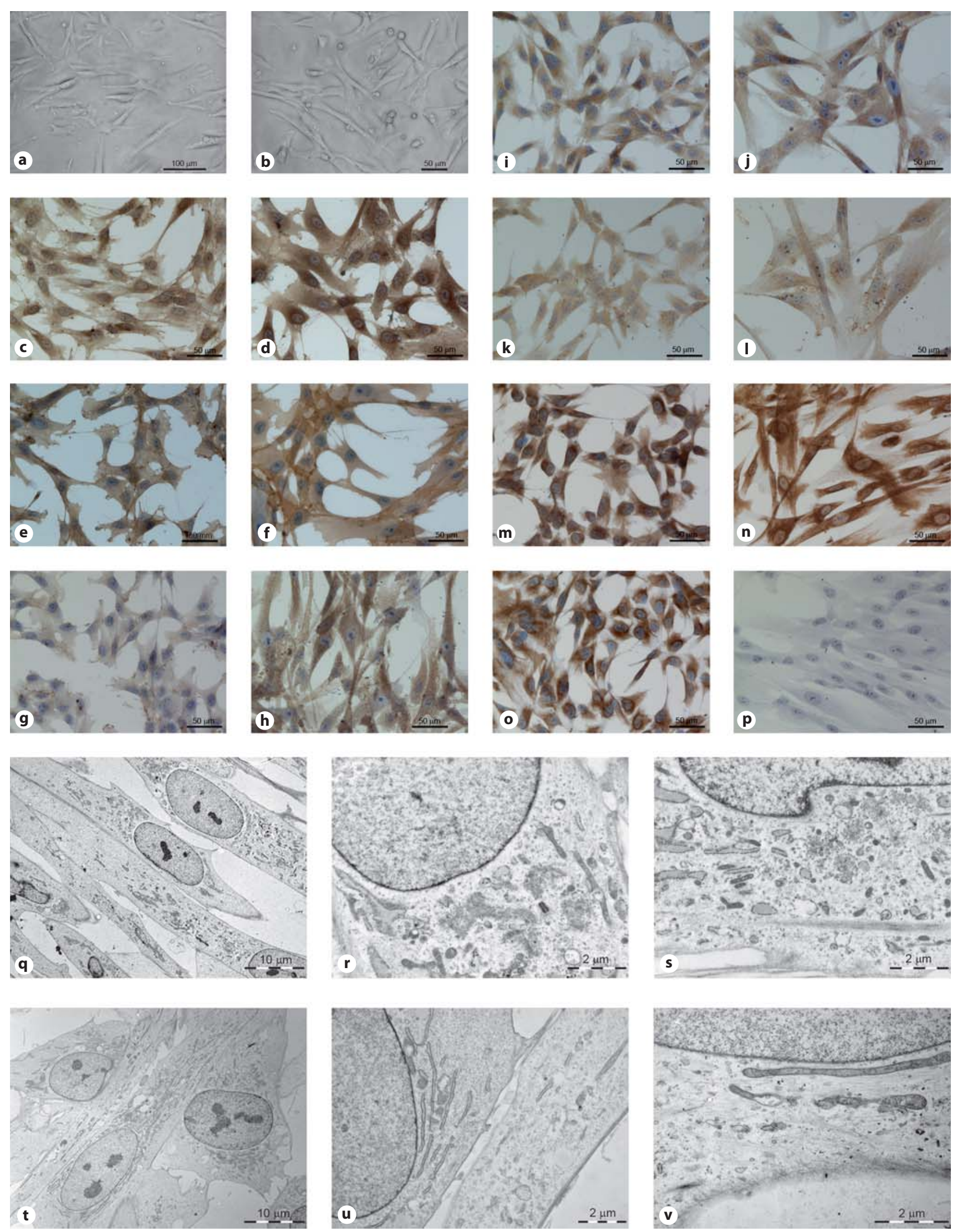

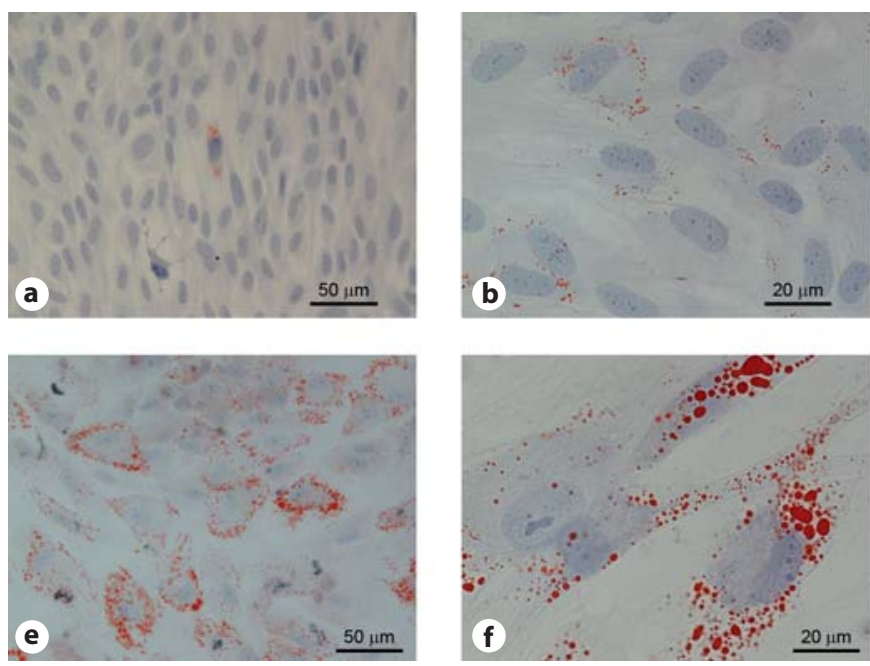

Fig. 2. Adipogenic differentiation of human DPSC (a-d) and UCSC (e-h) resulted in the accumulation of lipid droplets in the cell cytoplasm and the de novo expression of FABP. In contrast to UCSC (e, f), ORO staining did not always showed the presence of lipid droplets in human DPSC (a). Lipid droplet accumulation in the cytoplasm of human DPSC (b) could only be generated with
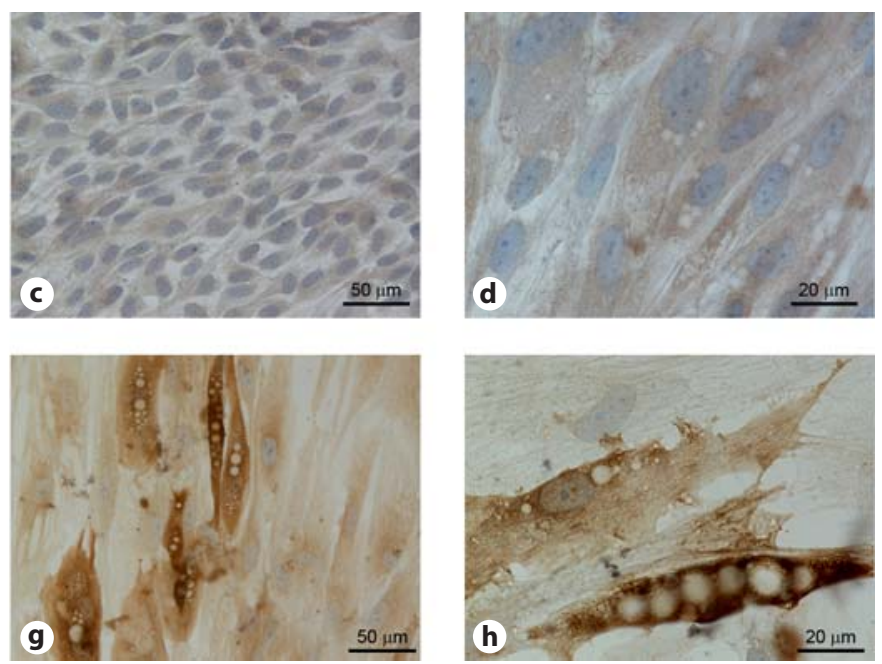

a $30 \%$ success rate. However, in both human DPSC (c) and UCSC (g), the de novo expression of FABP suggests differentiation into the adipogenic lineage. At a higher magnification, human DPSC (d) and UCSC (h) lipid droplet accumulation could be visualized in the cell cytoplasm. Note the difference in cell size between both differentiated cell types. tiny intracytoplasmic lipid droplets in fusiform-shaped cells. Shortly thereafter, most cells transformed into a round or more cuboidal shape and retracted their cellular extensions. Lipid granules tended to fuse, forming larger ones in the following weeks. The adipogenic differentiation of UCSC was highly efficient as ORO staining could be found in nearly $80-90 \%$ of the cells. However, the UCSC response to adipogenic induction was heterogeneous as there were differences in the number and size of the lipid droplets. The differentiation was confirmed de novo by the expression of FABP (fig. $2 \mathrm{~g}, \mathrm{~h}$ ). To evaluate the morphological features of the newly formed adipocytes, cells were examined by electron microscopy. Ultrastructural examination revealed a high accumulation of lipid droplets in the cytoplasm of differentiated cells (fig. 3c, d).

Osteogenic induction of DPSC and UCSC resulted in the occurrence of a calcified matrix which could be visualized using Alizarin red S staining. Nevertheless, the staining pattern within both cell type cultures was different. In the differentiated DPSC culture small, round, and dark, stained mineralized structures were present at different locations in the monolayer (fig. 4a). In contrast, the staining pattern in the differentiated UCSC culture seemed more diffuse and widespread (fig. 4c). In both cases, osteogenic differentiation resulted in the de novo ex- pression of osteocalcin (fig. 4b, d). Despite the difference at the light microscopical level, ultrastructural analysis of both differentiated cell types confirmed the acquisition of an osteoblastic phenotype. The cytoplasm of both cell types was characterized by the accumulation of multiple matrix vesicles (fig. 5a, b). Furthermore, both DPSC and UCSC secreted an extracellular matrix which mainly contained typically cross-banded collagen microfibrils (fig. $5 c, d$ ). However, only in the differentiated DPSC could multiple mineralization nuclei be visualized (fig. 5c). At a higher magnification, a globular secretion pattern and individual hydroxyapatite needles could be detected (fig. 5e). These mineralization nuclei tended to fuse with one another resulting in the presence of larger bone-like fragments which strongly interacted with the surrounding collagen (fig. 5f). These fragments also contained some open spaces resembling the lacunae of bone tissue.

Chondrogenic induction was investigated using highdensity cell pellet cultures in conditioned media to form 3D cell spheres. After 3 weeks, cell spheres consisted of a cell population residing in a heterogeneous extracellular matrix. Alcian blue staining of the cryosections of differentiated 3D cell pellets confirmed the production of glycosaminoglycans and glycoproteins (fig. 6a, b). In general, 3D cell spheres originating from differentiated DPSC were more densely organized compared to those 

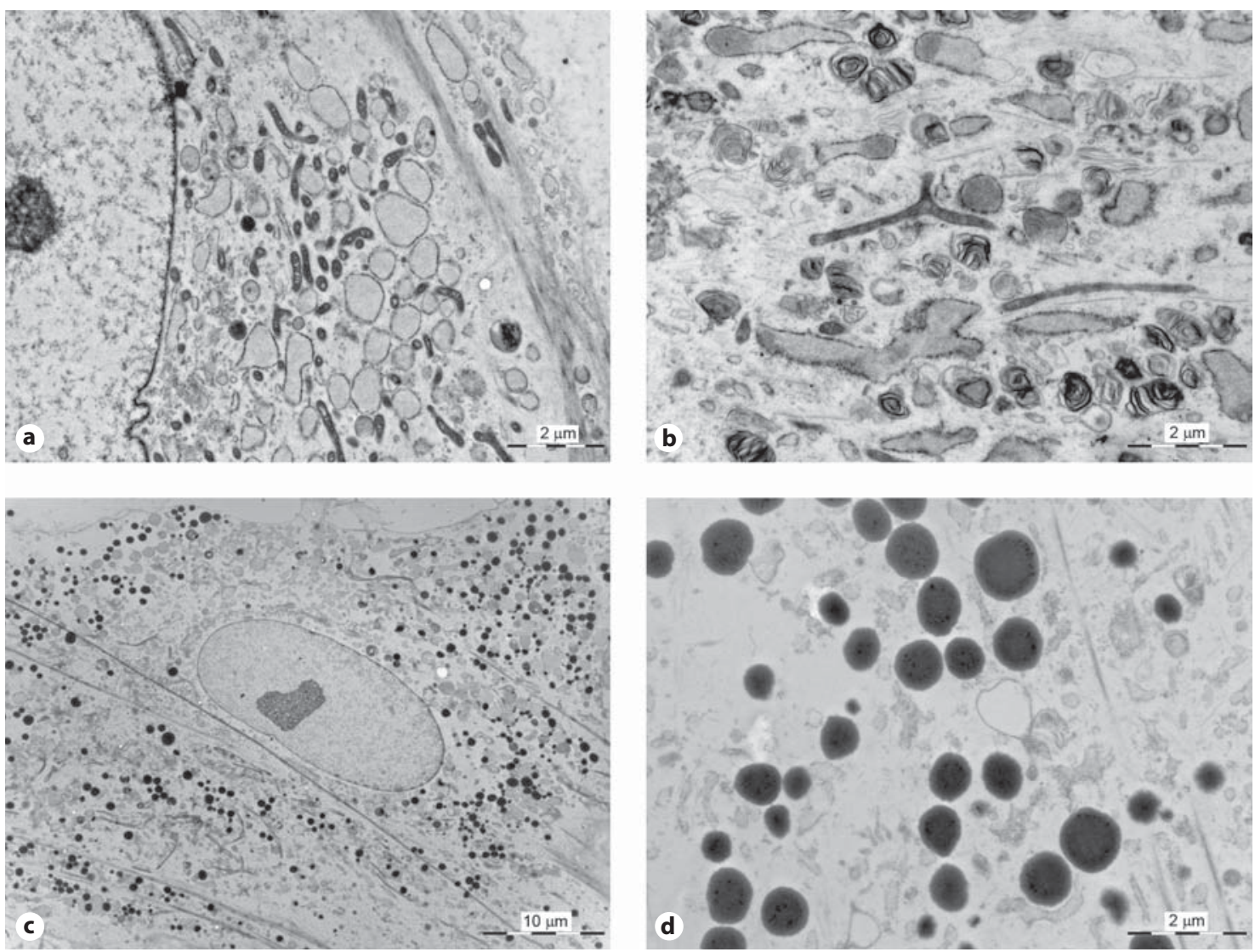

Fig. 3. Ultrastructural analysis of adipogenic-induced human DPSC (a, b) and UCSC (c, d). Ultrastructural examination by TEM demonstrated a high accumulation of lipid droplets in the cytoplasm of differentiated UCSC (c, d). In contrast, adipogenically-induced human DPSC (a, b) only showed an accumulation of multilamellar vesicles in the cytoplasm. Dilated RER and elongated and branched mitochondria were shared characteristics of the cytoplasmatic content of differentiated UCSC and human DPSC.

originating from differentiated UCSC. In both cases the 3D cell spheres were surrounded by a thin capsule consisting of long, elongated, fibroblast-like cells. Furthermore, differentiated human DPSCs and UCSC expressed aggrecan, a major structural protein of cartilage (fig. 6c, d). At a higher magnification, cartilage-like fragments could be detected in both cell types (fig. 6e, f). After 3 weeks, DPSC tended to produce more mature fragments in which a clear distinction between the territorial and interterritorial matrix was visible. Differentiation was confirmed at the ultrastructural level where prominent morphological changes could be described. Typically, cells acquired a chondrogenic phenotype which was mainly characterized by an accumulation of matrix vesicles in the cytoplasm and a massive production of collagen fibers (fig. 7a, d). Some vesicles were present in the extracellular space, strongly interacting with the sur- rounding collagen fibers. In addition, multiple cartilagelike fragments were present in the extracellular space which showed a close interaction with the surrounding chondroblasts (fig. 7e, h).

\section{Discussion}

The possibility of transplanting adult stem cells into damaged organs has opened new prospects for treating several human pathologies. Among the various possible sources of stem cells, MSCs have been studied extensively because of their ability to self-renew and to differentiate into cell types of mesodermal origin such as osteoblasts, chondrocytes, and adipocytes [Castro-Malaspina et al., 1980; Prockop, 1997; Pittenger et al., 1999; Barry and Murphy, 2004]. Recent studies have already shown 

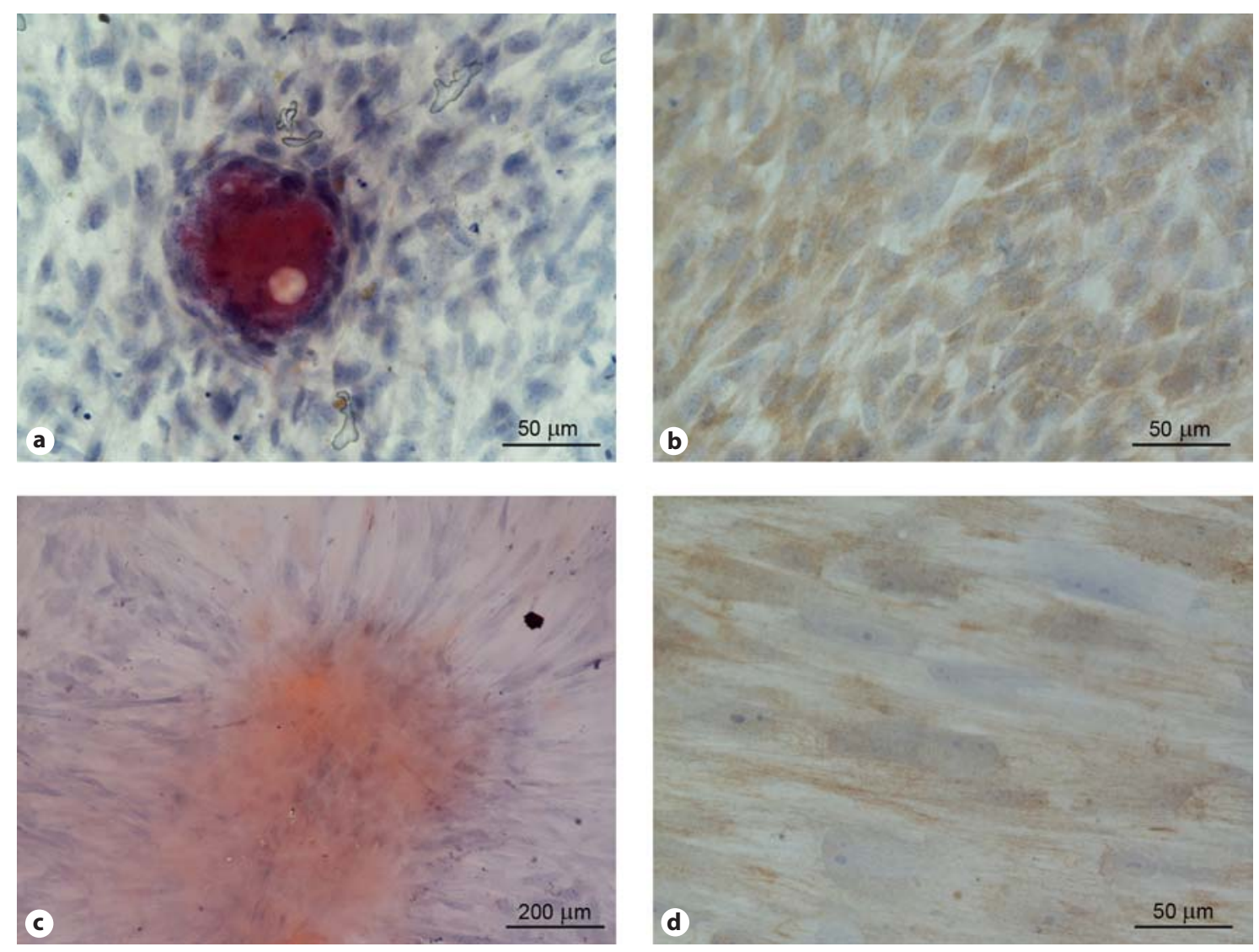

Fig. 4. Osteogenic differentiation of human DPSC (a, b) and UCSC (c, d) resulted in the presence of multiple mineralization nuclei in vitro which were visualized by Alizarin red S staining (a, $\mathbf{c})$. Furthermore, the de novo expression of osteocalcin could be detected following immunocytochemistry (b, d).

the MSC nature of cells within dental pulp and umbilical cord tissue, both classified as mucoid connective tissues [Gronthos et al., 2002; Mitchell et al., 2003; Weiss et al., 2006]. However, in-depth knowledge of their differentiation potential is essential in order to explore their usefulness for cell-based therapies. Although numerous researchers have performed genetic analyses of the cells, only a few manuscripts have been published concerning the morphological changes seen following MSC differentiation [Raimondo et al., 2006; Arminan et al., 2009; Karaoz et al., 2009]. This study was the first to examine the detailed process of the mesenchymal differentiation of stem cells using an electron microscope. We attempted to gain a better insight into the structural changes that 2 types of human MSCs undergo upon differentiation into mesenchymal lineages including adipogenic, chondrogenic, and osteogenic cell types. We believe this to be important in the field of stem cell biology because, although much is known concerning the biochemical changes a stem cell experiences during differentiation, little is known about the direct physical changes a cell goes through upon differentiation. This may have wide implications, especially in the field of tissue engineering where structural changes are as important as biochemical ones. Therefore, the present study provides a comprehensive morphological analysis of the differentiation capacities of DPSC and UCSC.

Both cell types were isolated according to the explant methodology that has been previously described for the isolation of UCSC [Mitchell et al., 2003; Conconi et al., 2006]. Isolated DPSC and UCSC were found to display a characteristic set of MSC markers including CD29, CD44, CD105, CD146, and STRO-1 [Gronthos et al., 2000; Mitchell et al., 2003; Weiss et al., 2006]. Furthermore, both cell types expressed vimentin, an intermediate filament found in cells of mesenchymal origin [Herrmann and Aebi, 2000]. In addition, DPSC were found to express neuroectodermal marker nestin, which consti- 

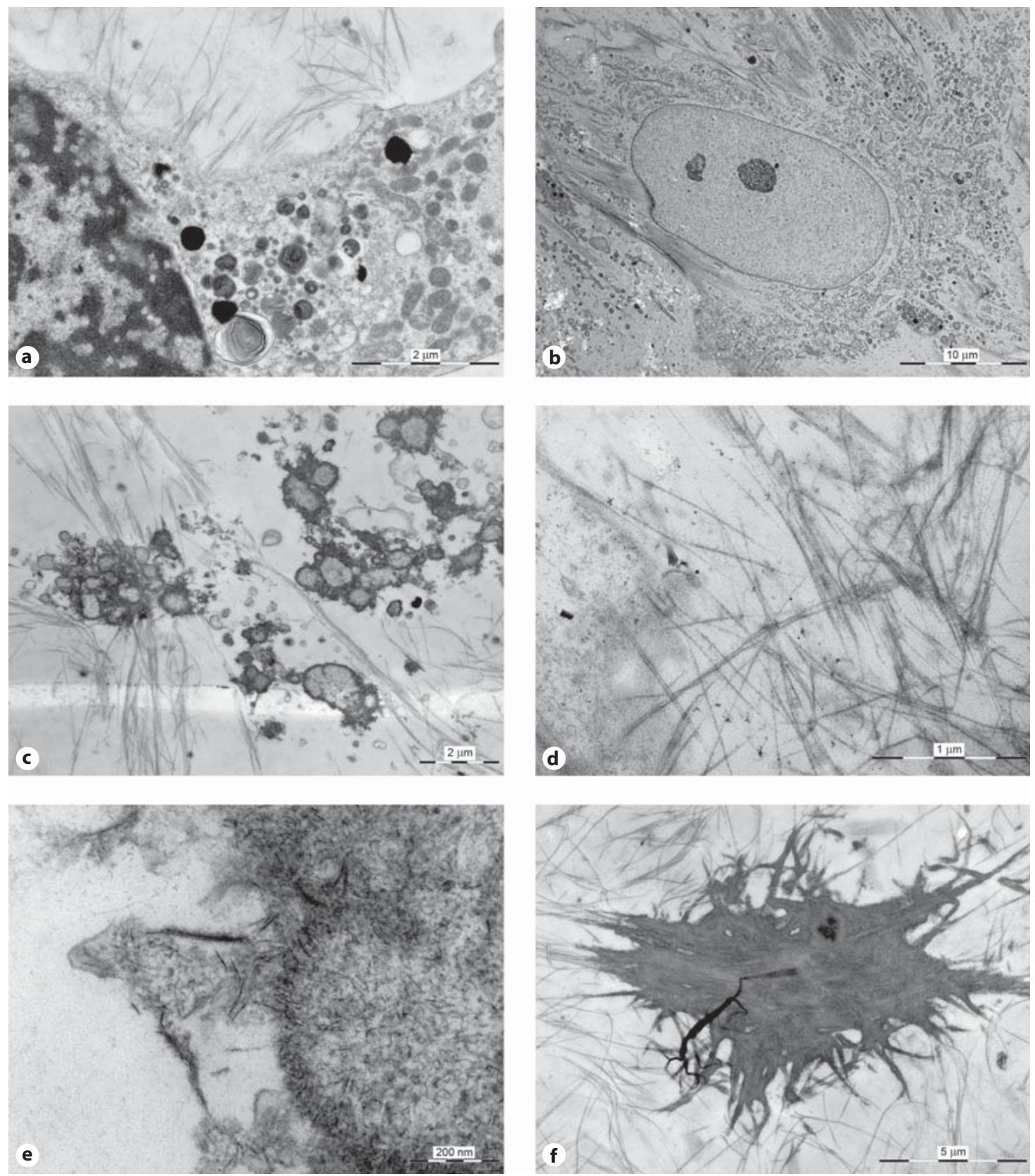

Fig. 5. Ultrastructural analysis of osteogenic-induced human $\operatorname{DPSC}(\mathbf{a}, \mathbf{c}, \mathbf{e}, \mathbf{f})$ and $\operatorname{UCSC}(\mathbf{b}, \mathbf{d})$. Characteristic for both cell types was the accumulation of matrix vesicles in the cytoplasm $(\mathbf{a}, \mathbf{b})$. Extracellular production of typically striated collagen fibers was present in both cell cultures (c, d). Only differentiated human
DPSC showed the presence of multiple mineralization nuclei (c) in which, at a higher magnification, the individual hydroxyapatite needles were visible (e). This process resulted in the presence of large bone-like fragments which strongly interacted with the surrounding collagen (f). tutes direct evidence of the neural crest origin of dental pulp [Terling et al., 1995]. In contrast, UCSC showed no expression of nestin. Currently, there is some controversy about the presence of nestin in UCSC [Mitchell et al., 2003; Fu et al., 2004; Ma et al., 2005; Weiss et al., 2006].
However, Wislet-Gendebien et al. [2005] demonstrated that nestin is only expressed in the absence of serum and at a minimum of 10 passages.

The pop-off technique was used to study both undifferentiated and differentiated cells. This technique allows 

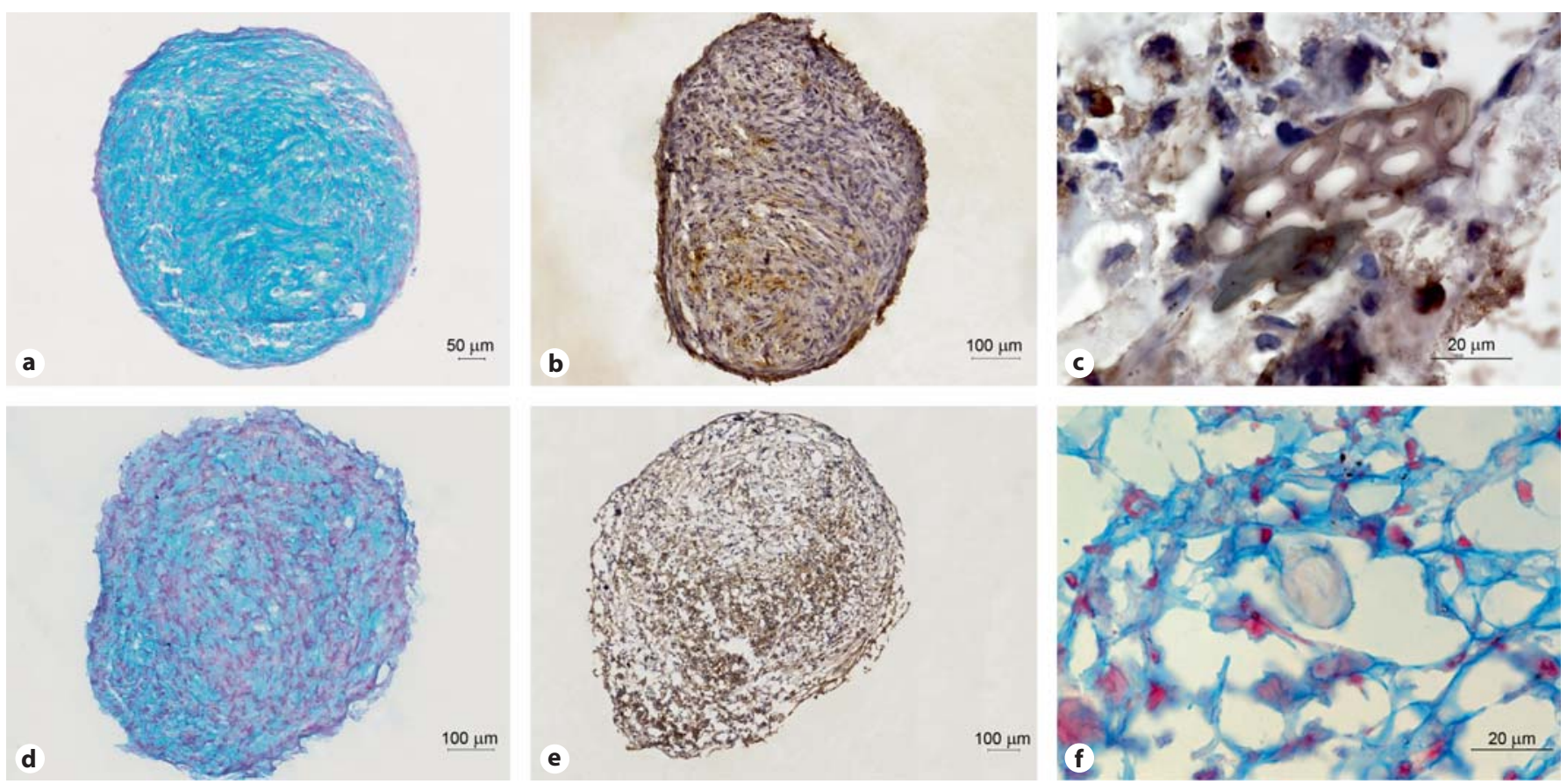

Fig. 6. Chondrogenic differentiation of human DPSC (a-c) and UCSC (d-f) was analyzed using Alcian blue staining (a, d). Differentiated human DPSC (a) seemed to produce more ground substance in comparison to differentiated UCSC (d). Nevertheless, differentiation resulted, in both cell types, in the de novo expression of aggrecan, a major structural protein of cartilage (b, e). At

a higher magnification, a hyaline cartilage-like fragment could be detected $(\mathbf{c}, \mathbf{f})$ in which a clear distinction between the territorial and interterritorial matrix could be made. The cultured cell spheres were surrounded by a thin capsule consisting of long, elongated, fibroblast-like cells which was very similar to the perichondrium in vivo.

the analysis of the ultrastructural characteristics of adherent cells. Our data showed that both cell types are relatively uniform, especially in terms of ultrastructure. In an undifferentiated state both cell types exhibited 2 distinct subcellular compartments comprising a perinuclear organelle-rich zone and a peripheral zone lacking organelles but characterized by the presence of intermediate filament bundles. Previous studies in which the ultrastructural characteristics of bone marrow MSCs were studied describe similar results [Raimondo et al., 2006; Karaoz et al., 2009]. However, these studies describe the ultrastructural characteristics of MSCs in suspension. To our knowledge, the present study describes for the first time the ultrastructural characteristics of adherent DPSC and UCSC.

Adipogenic differentiation of DPSC and UCSC with the same conditioned medium resulted in a different outcome. Although both cell types did express FABP following differentiation, ORO staining demonstrated some cells with lipid droplet accumulation only in $30 \%$ of the DPSC cultures. In contrast, all treated UCSC acquired an adipogenic morphology including positive ORO-stained lipid droplets. Moreover, in UCSC, lipid droplets were clearly visible at the ultrastructural level. Despite the absence of lipid droplet accumulation in treated DPSC, cells did seem to respond by the formation cytoplasmic multilamellar vesicles. Concerning DPSC, the described culture conditions seem to be inappropriate for complete adipogenic differentiation.

Both cell types produce an osteoid-like extracellular matrix containing cross-banded collagen microfibrils. At the ultrastructural level, DPSC show all stages of osteogenesis, starting with MSCs which develop into mature osteocytes. In addition, the secretory activity of differen-

Fig. 7. Ultrastructural analysis of chondrogenic-induced human $\operatorname{DPSC}(\mathbf{a}, \mathbf{c}, \mathbf{e}, \mathbf{g})$ and $\operatorname{UCSC}(\mathbf{b}, \mathbf{d}, \mathbf{f}, \mathbf{h})$. Ultrastructural analysis resulted in the accumulation of matrix vesicles in both cell types $(\mathbf{a}, \mathbf{b})$. Then, these vesicles tended to be secreted into the extracellular space where they started to fuse and interact with the surrounding collagen fibers (c-f). This resulted in the presence of hyaline cartilage-like fragments which seemed to grow by appositional secretion of the new chondrogenic matrix $(\mathbf{g}, \mathbf{h})$. 

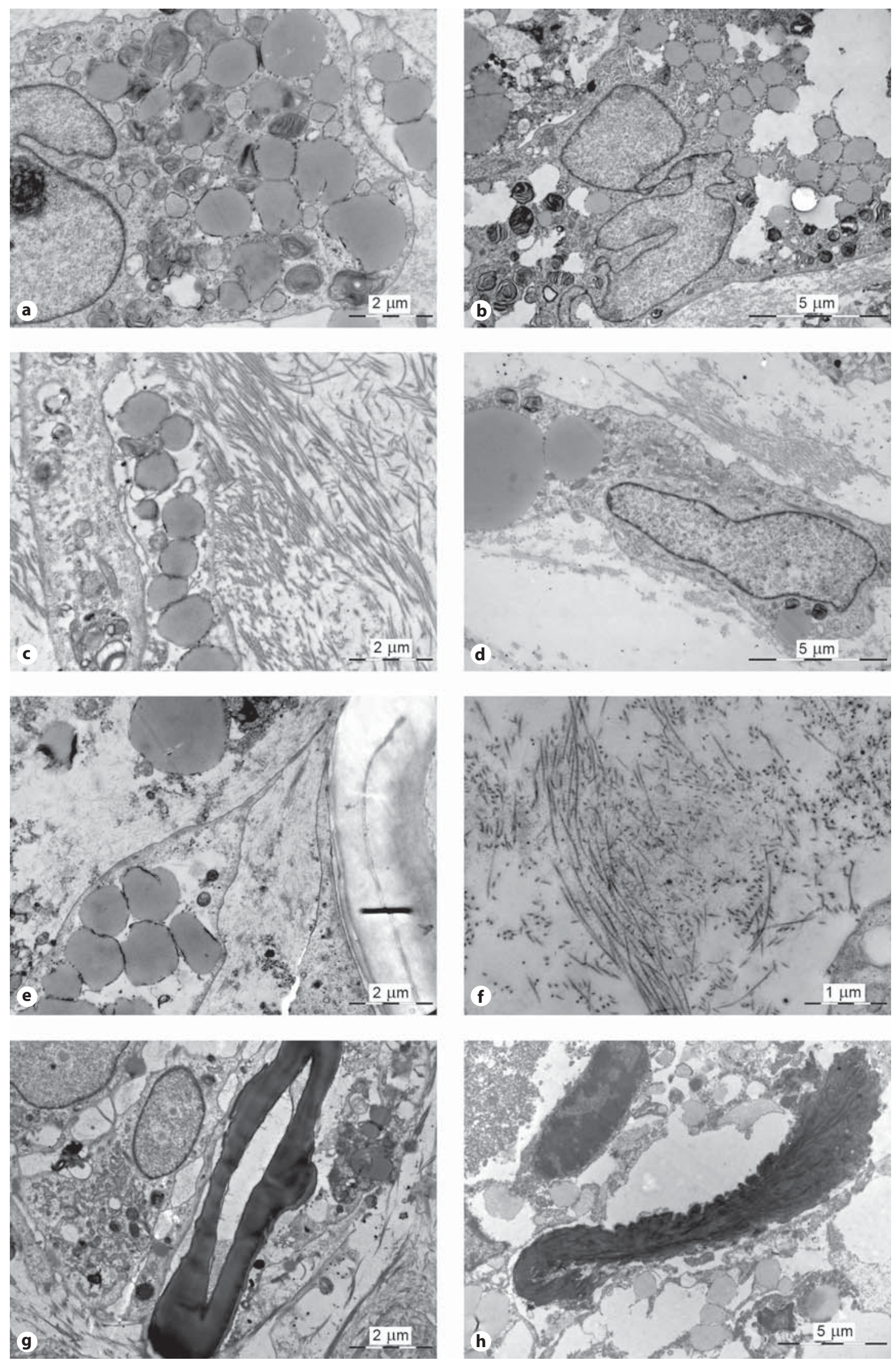
tiated DPSC very closely resembles the globular secretion pattern which is seen during in vivo osteogenesis [Yamamoto et al., 2005]. The presence of hydroxyapatite needles confirms the occurrence of real mineralization in the culture. In contrast, UCSC represent a more immature type of in vitro osteogenesis. This difference can be related to the environmental predisposition of the stem cells in both tissues of origin. DPSC have resided many years in a hard tissue environment which is likely to favor the cells' capacity to be induced into the osteogenic lineages. In contrast, UCSC have not been exposed to hard tissue development, which in turn could explain their better adipogenic induction. Nevertheless, our data confirm the osteoblastic potential of both cell types.

In vitro cartilage formation by MSCs is observed when cells are cultured as a micromass in a defined medium for chondrogenesis [Sekiya et al., 2001]. Cells located in the center of the 3D spheres showed many characteristics of metabolically active cells. Their cytoplasm contained numerous matrix vesicles. In addition, large amounts of proteoglycans and collagens filled the intercellular space between the cells. The homogeneous mass constituting the cartilage fragments suggests that chondrogenic cells produce typical hyaline cartilage as presents in joints and other tissues. In the cartilage fragments produced by differentiated DPSC, a clear distinction between the territorial and interterritorial matrix can be made. The close interaction of chondroblasts and cartilage fragments suggests that the latter expand by means of appositional growth.

In vitro mesenchymal tissue formation (bone, cartilage, and fat) by MSCs makes it possible to clarify the detailed morphological events that occur during osteogenesis, chondrogenesis, and adipogenesis, respectively. We emphasize that electron microscopical analysis is useful to elucidate detailed structural information. Moreover, ultrastructural changes provide indisputable evidence of differentiation.
Taken together, our results demonstrate that DPSC display at least bilineage differentiation potential, whereas UCSC, which are developmentally more primitive cells, show trilineage potential. A clonal study of hMSCs already described that only one third had osteo-, chondro-, and adipogenic potential, whereas $60-80 \%$ had osteo- and chondrogenic potential [Muraglia et al., 2000]. In this study practically all of the clones displayed osteogenic potential. Moreover, the study by Muraglia et al. [2000] showed that, upon culture, MSCs gradually lose their adipo- and chondrogenic potential. These results could explain the difference in the adipogenic potential of both cell types. Moreover, a recent study described the presence of 2 progenitor cell populations in dental pulp exhibiting different cellular characteristics in terms of their embryonic nature [Waddington et al., 2009]. This could also clarify the inability of DPSC to undergo adipogenesis in $100 \%$ of the cases because one of the progenitor cell populations might be more susceptible than the other.

In conclusion, the present study provides direct evidence that 2 MSC populations, distinct in their ontogeny and developmental potentials, are both plausible candidates for future use in cell-based therapies. Although DPSC and UCSC have much in common with bone marrow-derived MSCs, both cell types have distinct advantages in that they are easily obtained and are in abundant supply, thereby creating no donor site morbidity. As the present study was aimed at providing an in-depth morphological description of both DPSC and UCSC induced towards adipogenic, osteogenic, and chondrogenic lineages, we emphasize that electron microscopical analysis is useful to elucidate detailed structural information. Moreover, ultrastructural changes provide indisputable evidence of differentiation.

\section{References}

Arminan, A., C. Gandia, M. Bartual, J.M. Garcia-Verdugo, E. Lledo, V. Mirabet, M. Llop, J. Barea, J.A. Montero, P. Sepulveda (2009) Cardiac differentiation is driven by NKX2.5 and GATA4 nuclear translocation in tissuespecific mesenchymal stem cells. Stem Cells Dev 18: 907-918.

Barry, F.P., J.M. Murphy (2004) Mesenchymal stem cells: clinical applications and biological characterization. Int J Biochem Cell Biol 36: $568-584$.
Bretschneider, A., W. Burns, A. Morrison (1981) 'Pop-off' technic: the ultrastructure of paraffin-embedded sections. Am J Clin Pathol 76: 450-453.

Caplan, A.I. (2007) Adult mesenchymal stem cells for tissue engineering versus regenerative medicine. J Cell Physiol 213: 341-347.

Carlin, R., D. Davis, M. Weiss, B. Schultz, D. Troyer (2006) Expression of early transcription factors Oct-4, Sox-2 and nanog by porcine umbilical cord (PUC) matrix cells. Reprod Biol Endocrinol 4: 8.
Castro-Malaspina, H., R.E. Gay, G. Resnick, N. Kapoor, P. Meyers, D. Chiarieri, S. McKenzie, H.E. Broxmeyer, M.A. Moore (1980) characterization of human bone marrow fibroblast colony-forming cells (CFU-F) and their progeny. Blood 56: 289-301.

Conconi, M.T., P. Burra, R. Di Liddo, C. Calore, M. Turetta, S. Bellini, P. Bo, G.G. Nussdorfer, P.P. Parnigotto (2006) Cd105(+) cells from Wharton's jelly show in vitro and in vivo myogenic differentiative potential. Int J Mol Med 18: 1089-1096. 
Friedenstein, A.J., J.F. Gorskaja, N.N. Kulagina (1976) fibroblast precursors in normal and irradiated mouse hematopoietic organs. Exp Hematol 4: 267-274.

Fu, Y.S., Y.T. Shih, Y.C. Cheng, M.Y. Min (2004) Transformation of human umbilical mesenchymal cells into neurons in vitro. J Biomed Sci 11: 652-660.

Giordano, A., U. Galderisi, I.R. Marino (2007) From the laboratory bench to the patient's bedside: an update on clinical trials with mesenchymal stem cells. J Cell Physiol 211: 27-35.

Gronthos, S., J. Brahim, W. Li, L.W. Fisher, N. Cherman, A. Boyde, P. DenBesten, P.G. Robey, S. Shi (2002) Stem cell properties of human dental pulp stem cells. J Dent Res 81: 531-535.

Gronthos, S., M. Mankani, J. Brahim, P.G. Robey, S. Shi (2000) Postnatal human dental pulp stem cells (DPSCs) in vitro and in vivo. Proc Natl Acad Sci USA 97: 13625-13630.

Herrmann, H., U. Aebi (2000) Intermediate filaments and their associates: multi-talented structural elements specifying cytoarchitecture and cytodynamics. Curr Opin Cell Biol 12: 79-90.

Jiang, Y., B. Vaessen, T. Lenvik, M. Blackstad, M. Reyes, C.M. Verfaillie (2002) Multipotent progenitor cells can be isolated from postnatal murine bone marrow, muscle, and brain. Exp Hematol 30: 896-904.

Karaoz, E., A. Aksoy, S. Ayhan, A.E. Sariboyaci, F. Kaymaz, M. Kasap (2009) Characterization of mesenchymal stem cells from rat bone marrow: ultrastructural properties, differentiation potential and immunophenotypic markers. Histochem Cell Biol 132: 533-546.

Kobayashi, K., T. Kubota, T. Aso (1998) Study on myofibroblast differentiation in the stromal cells of Wharton's jelly: expression and localization of alpha-smooth muscle actin. Early Hum Dev 51: 223-233.

Lee, P.H., J.W. Kim, O.Y. Bang, Y.H. Ahn, I.S. Joo, K. Huh (2008) Autologous mesenchymal stem cell therapy delays the progression of neurological deficits in patients with multiple system atrophy. Clin Pharmacol Therapeutics 83: 723-730.
Linde, A. (1985) The extracellular matrix of the dental pulp and dentin. J Dent Res 64: 523 529.

Ma, L., X.Y. Feng, B.L. Cui, F. Law, X.W. Jiang, L.Y. Yang, Q.D. Xie, T.H. Huang (2005) Human umbilical cord Wharton's jelly-derived mesenchymal stem cells differentiation into nerve-like cells. Chin Med J 118: 1987-1993.

McElreavey, K.D., A.I. Irvine, K.T. Ennis, W.H McLean (1991) Isolation, culture and characterisation of fibroblast-like cells derived from the Wharton's jelly portion of human umbilical cord. Biochem Soc Trans 19: 29 S.

Mitchell, K.E., M.L. Weiss, B.M. Mitchell, P. Martin, D. Davis, L. Morales, B. Helwig, M Beerenstrauch, K. Abou-Easa, T. Hildreth, D. Troyer, S. Medicetty (2003) Matrix cells from Wharton's jelly form neurons and glia. Stem Cells 21: 50-60.

Mueller, S.M., J. Glowacki (2001) Age-related decline in the osteogenic potential of human bone marrow cells cultured in three-dimensional collagen sponges. J Cell Biochem 82: 583-590.

Muraglia, A., R. Cancedda, R. Quarto (2000) Clonal mesenchymal progenitors from human bone marrow differentiate in vitro according to a hierarchical model. J Cell Sci 113(pt 7): 1161-1166.

Pittenger, M.F., A.M. Mackay, S.C. Beck, R.K. Jaiswal, R. Douglas, J.D. Mosca, M.A. Moorman, D.W. Simonetti, S. Craig, D.R. Marshak (1999) Multilineage potential of adult human mesenchymal stem cells. Science 284 : 143-147.

Prockop, D.J. (1997) Marrow stromal cells as stem cells for nonhematopoietic tissues. Science 276: 71-74.

Raimondo, S., C. Penna, P. Pagliaro, S. Geuna (2006) morphological characterization of GFP stably transfected adult mesenchymal bone marrow stem cells. J Anat 208: 3-12.

Sekiya, I., D.C. Colter, D.J. Prockop (2001) BMP6 enhances chondrogenesis in a subpopulation of human marrow stromal cells. Biochem Biophys Res Commun 284: 411-418.

Shi, S., S. Gronthos (2003) Perivascular niche of postnatal mesenchymal stem cells in human bone marrow and dental pulp. J Bone Miner Res 18: 696-704.
Stenderup, K., J. Justesen, C. Clausen, M. Kassem (2003) aging is associated with decreased maximal life span and accelerated senescence of bone marrow stromal cells. Bone 33: 919-926.

Takeda, T., Y. Tezuka, M. Horiuchi, K. Hosono, K. Iida, D. Hatakeyama, S. Miyaki, T. Kunisada, T. Shibata, K. Tezuka (2008) Characterization of dental pulp stem cells of human tooth germs. J Dent Res 87: 676-681.

Terling, C., A. Rass, T.A. Mitsiadis, K. Fried, U. Lendahl, J. Wroblewski (1995) Expression of the intermediate filament nestin during rodent tooth development. Int J Dev Biol 39: 947-956.

Uccelli, A., V. Pistoia, L. Moretta (2007) Mesenchymal stem cells: a new strategy for immunosuppression? Trends Immunol 28: 219226 .

Waddington, R.J., S.J. Youde, C.P. Lee, A.J. Sloan (2009) Isolation of distinct progenitor stem cell populations from dental pulp. Cells Tissues Organs 189: 268-274.

Wang, H.S., S.C. Hung, S.T. Peng, C.C. Huang, H.M. Wei, Y.J. Guo, Y.S. Fu, M.C. Lai, C.C. Chen (2004) Mesenchymal stem cells in the Wharton's jelly of the human umbilical cord. Stem Cells 22: 1330-1337.

Weiss, M.L., S. Medicetty, A.R. Bledsoe, R.S. Rachakatla, M. Choi, S. Merchav, Y. Luo, M.S. Rao, G. Velagaleti, D. Troyer (2006) Human umbilical cord matrix stem cells: preliminary characterization and effect of transplantation in a rodent model of Parkinson's disease. Stem Cells 24: 781-792.

Weiss, M.L., K.E. Mitchell, J.E. Hix, S. Medicetty, S.Z. El-Zarkouny, D. Grieger, D.L. Troyer (2003) Transplantation of porcine umbilical cord matrix cells into the rat brain. Exp Neurol 182: 288-299.

Wislet-Gendebien, S., G. Hans, P. Leprince, J.M. Rigo, G. Moonen, B. Rogister (2005) Plasticity of cultured mesenchymal stem cells: switch from nestin-positive to excitable neuron-like phenotype. Stem Cells 23: 392-402.

Yamamoto, T., N. Nagaoka, A. Hirata, H. Nakamura, M. Inoue, M. Kawai, M. Ikegame (2005) Ultrastructural and immunohistochemical studies of medullary bone calcification, with special reference to sulphated glycosaminoglycans. J Electron Microsc 54: 29-34. 